\title{
Joseph und Marie-Anne Piller Preis
}

\author{
Julien Knebusch ${ }^{a}$, Alexandre Wenger ${ }^{b}$ \\ ${ }^{a}$ Lehr- und Forschungsrat, PhD, Universität Freiburg, Lehrstuhl für Medizin und Gesellschaft; ${ }^{b}$ Ordentlicher Professor, PhD, Universität Freiburg, Lehrstuhl \\ für Medizin und Gesellschaft
}

Im Rahmen des Programmes Medizin und Gesellschaft (Medical Humanities) der Universität Freiburg führen die Studierenden der Medizin im 3. Jahr alljährlich eine obligatorische Schreibübung durch: Nach einem 4-tägigen Praktikum bei einem Allgemeinmediziner verfassen sie einen detaillierten Bericht über eine Begegnung mit einem Patienten, der intensiv auf sie gewirkt hat. Der verfasste Text muss dabei bestimmte formale Kriterien bezüglich inhaltlicher Kohärenz der Darstellung, des Gesamtaufbaus oder auch der Relevanz der ausgewählten Informationen erfüllen. Auf diesen Text folgen dann einige Zeilen, in denen die beschriebene Situation noch einmal aufgenommen wird, diesmal aber in der Kurzform des medizinischen Berichtes: Der Kontrast zwischen beiden Schreibformen muss klar ersichtlich sein. Der Joseph und Marie-Anne Piller Preis zeichnet jeweils den besten französischsprachigen und deutschsprachigen Text aus.

Sich die Zeit nehmen und die Studierenden der Medizin zum Schreiben eines persönlichen Textes bewegen ja, eine solche Übung ist in einem kollektiv ausgerichteten, «kreuzlastigen» und von einem Wissensmarathon geprägten Studium erst einmal gewöhnungsbedürftig. Dennoch handelt es sich um eine fundamentale Übung für unsere späteren Ärzte. Schreiben bedeutet, die eigenen Worte sorgfältig auszuwählen, seine Gedanken zu ordnen, sich dem Adressaten dieser Worte bewusst zu werden und die eigenen Voraussetzungen zu beherrschen. Die Schreibübung ermöglicht dem Studierenden, die Begegnung mit einem Patienten in ihrer gesamten Komplexität zu erfassen, indem er über den Weg der Schilderung einen reflexiven Abstand zum Geschehenen nehmen kann. Das beschwerliche Medizinstudium und der sich oft mühsam aneinanderreihende medizinische Alltag machen eine solche "Atempause» mehr als notwendig.

Sprache und deren Gebrauch sind ein Schlüsselelement für die Kommunikation mit dem Patienten und die klinische Praxis. Vom mechanischen Erlernen von Empathie weit entfernt, bringt die Schilderung einer Begegnung mit einem Patienten den Studierenden dazu, diesen Augenblick in seiner Gesamtheit zu erfassen. Er wird mit der Vielfalt an kulturellen und sozialen Begebenheiten konfrontiert, welche diese Begegnung unter Verwendung des wunderbaren Mittels der Sprache beleuchten können. Leider werden Sprache und Sprachgebrauch in unseren medizinischen Studiengängen nach wie vor stiefmütterlich behandelt. Dabei beobachten wir doch ebenso mit Worten ... oder etwa nicht?

Die Schreibübung ermöglicht dem Studierenden, die Begegnung mit einem Patienten in ihrer gesamten Komplexität zu erfassen, indem er über den Weg der Schilderung einen reflexiven Abstand zum Geschehenen nehmen kann.

Die Schreibübung schafft somit einen Raum zur Reflexion. Sie ermöglicht den Studierenden, sich hinsichtlich der erworbenen Kenntnisse zu positionieren. Sie führt dazu, dass eigene Grenzen sowie Formulierungsschwierigkeiten, welche die therapeutische Beziehung beeinträchtigen können, erkannt werden, und legt andererseits die viel zu oft ignorierte Fülle sprachlicher Ressourcen offen.

Nach zweifacher Durchsicht aller 115 in diesem Jahr erhaltenen Texte wurden die drei besten deutschsprachigen Texte anonymisiert und an eine Jury überreicht, die sich aus Anja Dirks (Leiterin des Festivals Belluard Bollwerk International), Alan Niederer (Neue Zürcher Zeitung, Wissenschaftsredaktion) und Peter Suter (ehemaliger Präsident Schweizerische Akademie für Medizinwissenschaft) zusammensetzte. Am Ende dieses Verfahrens wurde schliesslich ein Text ausgewählt, den wir Ihnen an dieser Stelle vorstellen dürfen: «Wie zwei Freunde» von Benedikt Locher. Die Juroren schätzten insbesondere die Qualität des sprachlichen Ausdrucks, den Aufbau des Textes sowie die Richtung des Handlungsverlaufes, die dem Text Spannung verleiht und für ein unterhaltsames Leseerlebnis sorgt.

\section{Bemerkung}

In der französischsprachigen Ausgabe der Schweizerischen Ärztezeitung wird diese Woche der beste französischsprachige Text, verfasst von Mick Rosset, veröffentlicht. 